(C) Elsevier/INRA

Article original

\title{
Application de la cryoconservation des embryons à la protection des ressources génétiques chez le lapin
}

\author{
T Joly ${ }^{1,2}, \mathrm{M}$ Theau-Clément ${ }^{3}, \mathrm{~F}$ Drouet-Viard ${ }^{4}$, \\ $\mathrm{H}$ de Rochambeau ${ }^{3}$, JP Renard ${ }^{1}$
}

1 Institut national de recherche agronomique, unité de biologie du développement, 78352 Jouy-en-Josas, Cedex;

2 ISARA, 31, place Bellecour, 69002 Lyon Cedex;

${ }^{3}$ Institut national de recherche agronomique, station d'amélioration génétique des animaux, Auzeville BP 27, 31326 Castanet-Tolosan Cedex;

4 Institut national de recherche agronomique, unité de pathologie du lapin, 37380 Nouzilly, France

\begin{abstract}
Résumé - L'établissement d'une cryobanque de gamètes et d'embryons peut constituer aujourd'hui une aide précieuse pour garantir le maintien de la diversité génétique animale et la protection des populations menacées d'extinction. Nous avons entrepris d'établir une telle banque chez le lapin, espèce à la fois d'intérêt zootechnique, mais aussi modèle pour la recherche biomédicale. Dans un premier temps, nous avons défini les conditions techniques pour l'établissement d'une banque d'embryons à partir de la souche en cours d'homogénéisation «hyperféconde» de l'INRA, choisie comme référence. Nous avons ensuite commencé à appliquer ces techniques à la cryoconservation de plusieurs types de populations : une souche à grand effectif et de haute valeur zootechnique, la souche INRA A 1077, sélectionnée depuis 18 générations sur la taille de portée et maintenue en population fermée; une race à effectif réduit, le «Brun Marron de Lorraine»; une lignée d'intérêt biomédical constituée par 3 familles sélectionnées sur la variabilité allotypique des gènes codant pour la chaîne lourde des immunoglobulines; enfin, une lignée mutante, le lapin dit «sauteur d'Alfort». Cette recherche devrait nous permettre de définir les choix scientifiques et économiques qui présideront à l'extension de ce travail de conservation à d'autres populations de lapin, notamment des lignées transgéniques et des clones.
\end{abstract}

conservation / diversité génétique / cryobanque / lapin

Summary - Cryopreservation of embryos applied to the protection of genetic resources in the rabbit. The establishment of a cryobank of gametes and embryos would be useful for maintaining animal genetic diversity and protecting populations threatened with extinction. We have begun to establish such a bank using the rabbit as a model, a 
species which is of interest from both zootechnical and biomedical points of view. We first defined the technical parameters for establishing an embryo bank using the INRA 'hyperfecund' line. Subsequently we have begun to apply these techniques of cryopreservation to the following populations : a strain with a large population size and high zootechnical value, INRA A-107\%, selected over 18 generations for litter size and maintained as a closed breeding group; a breed with reduced population size, the 'Brun-marron de Lorraine'; a line of biomedical interest consisting of 3 families selected for allotypic variation in genes coding for the immunoglobin heavy chain; and a mutant strain referred to as the 'jumping Alfort' rabbit. This research should allow us to define the scientific and economic choices important in the extension of this work to other rabbit populations, particularly transgenic lines and clones.

conservation / genetic diversity / cryobank / rabbit

\section{INTRODUCTION}

Chez les animaux domestiques, l'utilisation intensive d'un petit nombre de races se traduit par un appauvrissement de la variabilité génétique des populations souvent issues de longs efforts de sélection et menace les races de petits effectifs (FAO, 1987). La définition de stratégies de conservation devient un impératif que la recherche ne peut ignorer ne serait-ce que pour corriger des erreurs commises parfois avec ou en son nom.

Dans le même temps, chez les animaux dits de laboratoire (la souris surtout), des outils comme la transgenèse associée maintenant à la mutagenèse insertionnelle ciblée permettent de créer de nouvelles lignées qui deviennent autant de modèles irremplaçables pour l'avancée des connaissances fondamentales et leurs applications à des fins biomédicales. Associés aux progrès en cours dans l'établissement des cartes génétiques et aux méthodes d'introgression de gènes dans une population, ces outils seront demain utilisés chez plusieurs espèces domestiques.

Dans les 2 situations, la conservation d'une diversité génétique menacée ou foisonnante apparaît comme indispensable pour ouvrir de nouveaux champs à la meilleure utilisation possible de caractères phénotypiques qui résultent le plus souvent de l'expression de plusieurs gènes combinants leurs effets au sein du génome.

Notre travail vise à fournir chez une espèce domestique prise comme modèle, le lapin (Oryctolagus cuniculus), une base de références concrètes pour la mise en place, l'organisation et l'utilisation de méthodes de conservation ex situ destinées à renforcer les actions indispensables de conservation in situ déjà à l'œuvre chez cette espèce et dont le développement doit être activement poursuivi. Une des méthodes de choix pour la conservation ex situ consiste à placer des embryons et des gamètes dans l'azote liquide à la température de $-196^{\circ} \mathrm{C}$ puisque l'on a montré qu'à cette température on pouvait conserver des spermatozoïdes bovins pendant plus de 30 ans et des embryons de souris pendant plus de 13 ans sans modifier leur aptitude à donner des descendants normalement fertiles (Wood et al, 1987). Ces durées seront probablement allongées au fur et à mesure que l'on disposera du recul expérimental suffisant. En effet, à la température de $-196^{\circ} \mathrm{C}$, seules des réactions photophysiques peuvent se dérouler, celles-ci risquant d'induire une ionisation du milieu cellulaire par accumulation de radiations de faibles niveaux. Or, des expériences simulant 
cet effet par l'exposition d'embryons de souris à l'équivalent d'environ 2000 années de radiations n'ont eu aucune conséquence génétique sur les souris issues de ces embryons après transplantation dans des femelles receveuses (Glenister et al, 1984), ce qui indique que la durée de conservation n'a dans ces conditions aucun effet néfaste sur les cellules.

Plus d'une centaine de races et variétés de lapin ont été à ce jour inventoriées par la World Rabbit Science Association pour une population mondiale estimée à plus de 700 millions d'individus (Lukefahr, 1985). Cet inventaire encore très partiel montre qu'on peut accéder avec cette espèce à des populations constituées de façons très différentes. À la diversité des situations génétiques rencontrées, le lapin joint un autre avantage. Chez cette espèce, des méthodes de congélation ont déjà été définies pour 2 types cellulaires, les spermatozoïdes et les embryons; l'insémination artificielle avec du sperme congelé peut être utilisée en pratique (Courot, 1977; Theau-Clément et Vrillon, 1989) et les techniques de cryopréservation d'embryons (Whittingham et Adams, 1976) sont compatibles avec des taux de développement à terme similaires à ceux obtenus en l'absence de congélation (Renard et al, 1984).

\section{ÉTABLISSEMENT D'UNE CRYOBANQUE D'EMBRYONS : DONNÉES TECHNIQUES}

L'utilisation des basses températures pour la préservation ex situ du génome des mammifêres domestiques a fait l'objet de nombreux travaux. Les auteurs formulent des recommandations parfois précises (FAO, 1987) mais qui s'appuient sur des données expérimentales partielles. Il est en effet largement fait appel à des considérations théoriques ou à des situations modèles qui empruntent à plusieurs espèces à la fois les données nécessaires à l'établissement de stratégies de conservation (Brem et al, 1982; Renard et al, 1983; Smith, 1984; Yamada et Kimura, 1984). La première partie de notre travail a donc consisté à mesurer les paramètres techniques conditionnant la mise en œuvre d'une cryobanque d'embryons de lapin. Nous avons choisi comme population de référence la souche $n^{\circ} 1029$ de l'INRA. Cette souche est utilisée pour l'étude génétique des composantes physiologiques de la taille des portées (Bolet et al, 1991).

\section{Méthode de congélation retenue}

Nous avons pris le parti de recourir à des techniques de stimulation hormonale et d'insémination artificielle pour disposer de références sur l'apport de ces méthodes à la constitution d'une banque d'embryons. La technique de superovulation décrite par Kennelly et Foote (1965) a été modifiée de la façon suivante : une dose de $2 \mathrm{mg}$ de pFSH (Stimufol, Rhône Mérieux, France) est administrée par voie sous-cutanée en 5 injections à $12 \mathrm{~h}$ d'intervalle (respectivement 0,$25 ; 0,25 ; 0,625 ; 0,625$ et $0,25 \mathrm{mg}$ ); $8 \mathrm{~h}$ après la dernière injection, les femelles sont inséminées (Theau-Clément et Roustan, 1991), avant induction de l'ovulation par un analogue de GnRH $(0,2 \mathrm{ml}$ de Buséréline en intramusculaire; Réceptal, Distrivet, France); les embryons sont collectés au stade morula $65 \mathrm{~h}$ post coïtum après abattage des femelles et perfusion du tractus génital à l'aide d'une solution saline tamponnée (tampon phosphate, PBS). Une technique délicate de collecte in vivo définie au laboratoire il y a quelques 
années (Garnier et al, 1988) et qui permet de récupérer les embryons plusieurs fois de suite sur une même femelle n'a pas été retenue pour ce premier travail. Elle le sera ultérieurement pour définir les conditions strictes de son application dans le cas où le nombre de femelles de la population à conserver est très faible.

La méthode de congélation retenue implique l'emploi d'un congélateur programmable puisqu'elle est réalisée dans des conditions d'équilibre de variation de la température (Mazur, 1990). Le dispositif que nous utilisons est portable (Cryocell 1200. IMV, L'Aigle France) et son maniement simple en fait un appareil bien adapté pour intervenir directement dans des élevages. La méthode employée comprend 3 étapes. La première consiste à remplacer une partie de l'eau intracellulaire par une solution de cryoprotecteur; pour cela, les embryons sont plongés chaque fois pendant 5 minutes dans 3 bains successifs contenant respectivement $0,5,1,0$ et 1,5 M de di-méthyl sulfoxide (DMSO), avant d'être introduits dans une paillette en plastique du type de celles utilisées dans l'industrie de l'insémination artificielle bovine $(0,25 \mathrm{ml}$; IMV) mais modifiées pour permettre une identification sur un jonc de plastique. Dans un $2^{\mathrm{e}}$ temps, les paillettes sont équilibrées à $-7^{\circ} \mathrm{C}$ pendant 10 minutes dans le congélateur programmable prérefroidi; cette opération permet de mettre le milieu environnant les cellules en surfusion et favorise l'apparition d'un premier cristal de glace. La troisième étape consiste alors à abaisser lentement la température à une vitesse de $0,5^{\circ} \mathrm{C}$ par minute jusqu'à $-30^{\circ} \mathrm{C}$, ce qui permet une déshydratation progressive mais suffisante des cellules avant leur immersion dans l'azote liquide à $-196^{\circ} \mathrm{C}$. La décongélation s'effectue en réchauffant le plus rapidement possible les paillettes pour éviter la recristallisation éruptive de la glace intracellulaire. Le plus simple pour cela est de les faire passer directement de l'azote liquide dans un bain-marie à $20^{\circ} \mathrm{C}$. Le cryoprotecteur est retiré en plaçant les embryons successivement pendant 5 minutes dans 2 bains contenant 1,0 puis $0,5 \mathrm{M}$ de DMSO. La survie des embryons est mesurée par le taux de développement obtenu soit après $24 \mathrm{~h}$ de culture (taux d'embryons au stade blastocyste «épanoui») soit par transfert chirurgical dans les cornes utérines ou oviductes d'une femelle pseudogestante.

\section{Un premier bilan}

Soixante-quinze femelles âgées de 4,5 mois et élevées dans les mêmes conditions d'environnement ont été utilisées comme donneuses $(n=40)$ et receveuses $(n=35)$ d'embryons. Après application d'un traitement de superovulation, nous avons congelé en moyenne 11,5 embryons par femelle traitée. La variabilité de réponse au traitement est élevée. Plus du quart des animaux (11/40, soit $27,5 \%)$ ne donnent aucun embryon congelable; les autres fournissent en moyenne 15, $9 \pm 13$ embryons par individu. Le bilan de la congélation est évalué in vivo à partir du taux de développement à terme des embryons (après transfert dans des receveuses synchronisées) ou in vitro après $24 \mathrm{~h}$ de culture (tableau I).

Près de $90 \%$ des embryons congelés sont intacts à la décongélation et plus de $60 \%$ de ces embryons cultivés in vitro ou transférés sont capables de se développer et donner naissance à des lapereaux viables.

La distribution des donneuses en fonction du nombre de descendants obtenus est présentée sur la figure 1 . 


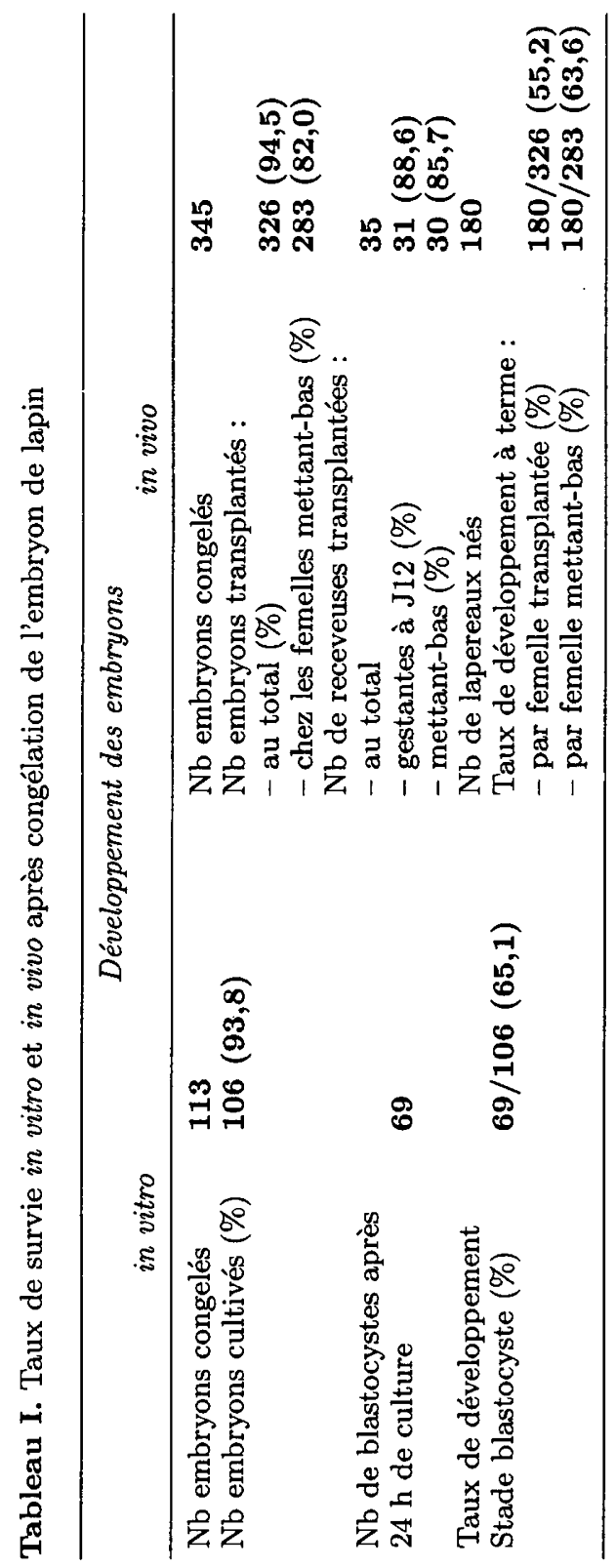




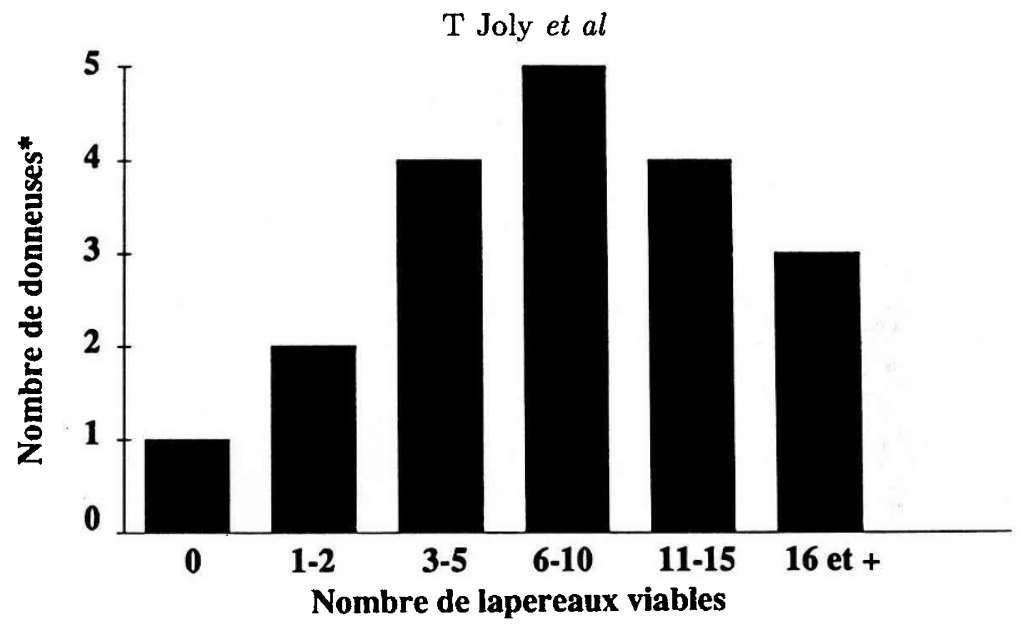

Fig 1. Distribution des donneuses d'embryons en fonction du nombre de leurs descendants. * Femelles qui ont donné au moins 9 embryons congelés et transplantés dans des receveuses.

Ces résultats montrent que sur les 19 femelles qui ont donné au minimum 9 embryons congelés et transplantés, $18(94,7 \%)$ d'entre elles ont eu au moins 1 descendant viable et $7(36,8 \%)$ plus de 10 lapereaux, soit une moyenne de 9,5 descendants par femelle (180/19). En définitive, ces différents éléments nous permettent d'en déduire qu'après congélation d'au moins 9 embryons, plus de $80 \%$ des accouplements (16/19) donnent 3 descendants viables ou plus ce qui, compte tenu d'une mortalité entre la naissance et la puberté d'environ $20 \%$, est compatible avec l'obtention d'un couple de reproducteurs adultes.

Des essais sont en cours pour confirmer ces premiers résultats et définir des paramètres techniques plus précis pour l'établissement de protocoles d'échantillonnage et de gestion des accouplements des animaux donneurs d'embryons en prenant en compte la variabilité individuelle de réponse des femelles. Trois paramètres techniques permettront alors de déterminer le nombre d'embryons qui doivent être congelés :

- la probabilité pour un couple donné de reproducteurs d'obtenir au moins un descendant de chaque sexe; ce paramètre dépend directement de l'efficacité technique de la cryoconservation;

- le nombre d'animaux retenus pour représenter génétiquement la population à conserver; ce nombre dépend des objectifs génétiques visés et du coût de la conservation;

- le plan d'accouplements nécessaires pour pouvoir réaliser un échantillonnage correct de la population. On estime que pour une population d'effectif réduit, de quelques centaines d'individus seulement, l'échantillon doit représenter au minimum 20 accouplements de parents le moins apparentés possible (Woodford, 1990). Pour ces populations, l'objectif est de conserver le maximum de produits de différents accouplements. Pour les populations de plusieurs milliers d'individus, des techniques d'échantillonnage permettent d'organiser les accouplements pour maintenir la plus large représentativité du pool génétique. 


\section{CHOIX DE POPULATIONS DE RÉFÉRENCE}

Dans le cadre de l'action que nous conduisons, 4 races (ou souches) font l'objet d'un programme de conservation. Chacune correspond à un type de population différent.

\section{Souche à grand effectif possédant une haute valeur zootechnique : la souche $A 1077$ de l'INRA}

Cette population est un bon exemple des races à grands effectifs (plusieurs milliers d'animaux). Les animaux sont utilisés pour leur aptitude à la production de viande (principalement les races néo-zélandaise et californienne) ou de fourrure (races Rex, Angora). Certaines souches issues de ces races font l'objet de plans d'amélioration génétique rigoureux (Matheron et Poujardieu, 1984). C'est le cas de la souche A 1077. Celle-ci, d'origine néo-zélandaise blanche, est sélectionnée artificiellement dans les installations de la station INRA de Toulouse depuis 1976 pour améliorer la taille de la portée au sevrage. Les animaux disponibles aujourd'hui sont les descendants de 11 mâles et 53 femelles fondatrices. La souche fermée dès l'origine est à sa vingtième génération de sélection. Elle est conduite en 11 groupes de reproduction formés en pratique de 4 mâles pleins frères et de 11 femelles. La valeur des coefficients de consanguinité est connue; à la dix-huitième génération, elle était de $0,144 \pm 0,037$ chez les mâles et $0,153 \pm 0,041$ chez les femelles (Poujardieu, communication personnelle). La sélection se poursuit selon un plan d'accouplements précis (Matheron et Chevalet, 1977) qui vise à distribuer l'ensemble des gènes sur l'ensemble des individus et donc à éviter que ne se constituent des pseudo-isolats génétiques. L'échantillon des reproducteurs utilisés pour produire les embryons peut dans ces conditions correspondre aux accouplements tels qu'ils sont prévus dans la gestion normale de la souche puisque, pour une génération donnée, on peut considérer que tous les reproducteurs qui donneront des futurs reproducteurs sont des fondateurs. La conservation sous forme d'embryons congelés vise d'abord à constituer une réserve de gènes disponibles en cas de catastrophe (problèmes sanitaires sur la population de base, destruction de l'élevage par incendie...). Mais elle constitue aussi un témoin pour estimer le progrès génétique réalisé sur une période de 5 ou 10 ans.

\section{Race à effectif réduit : le «Brun-marron de Lorraine»}

À côté des «grandes races» directement exploitées pour une production, on trouve un grand nombre de races possédant des qualités sans valorisation économique directe, isolées par des éleveurs passionnés attachés le plus souvent à des caractères de phanères (aspect du pelage, coloration particulière). Ces populations d'une centaine d'animaux sont souvent menacées d'extinction. Dans un pays comme la France, il en existe actuellement encore une quarantaine bien caractérisées par référence à un standard, et qui forment des populations de petits effectifs maintenus in situ chez les éleveurs (Rochambeau et Vrillon, 1980). La plupart d'entre elles ne sont constituées que d'environ 200 femelles et 20 mâles adultes, ce qui justifie la mise en œuvre d'un plan de sauvegarde adapté (Maijala, 1984). Selon Lukefahr (1990) un tel effort n'est plus utile à partir du moment où la race compte moins de 25 animaux 
sur une période consécutive de 5 ans, l'intérêt par les éleveurs collectionneurs étant alors manifestement insuffisant.

La race que nous avons retenue comme premier modèle pour la conservation de ce type de population est le Brun-marron de Lorraine. C'est une race légère d'origine française, créée au début du siècle par C Kauffmann et obtenue par croisement du Garenne et du Noir et Feu. Ces lapins de petite taille ont un poids moyen adulte de 1,5 à $2 \mathrm{~kg}$, une conformation et une coloration de pelage dérivée du lapin de garenne. L'intérêt principal de cette race réside dans sa bonne adaptation à des conditions précaires d'élevage (température, microbisme). Mais face au peu d'intérêt actuel des éleveurs français, elle n'est plus représentée que par à peine une centaine d'individus. En accord avec la Fédération française de cuniculture, l'objectif est de conserver des embryons en complément d'animaux vivants. L'échantillon à conserver est constitué de 10 familles issues d'élevages différents et comprenant chacune un mâle et 2 femelles. Le coefficient de parenté entre individus des différentes familles est considéré comme pratiquement nul. Le schéma de circulation des mâles fondateurs utilisés pour l'accouplement a été décrit par Rochambeau et Chevalet (1989). Les embryons collectés sur les femelles de deuxième génération constituent donc le stock représentant au maximum la variabilité génétique actuelle de la race Brun-marron de Lorraine.

\section{Une lignée d'intérêt potentiel pour la recherche fondamentale : les lapins porteurs d'allotypes rares}

Les lignées utilisées pour la recherche fondamentale et biomédicale sont maintenues le plus souvent en petits effectifs de quelques dizaines d'individus. C'est le cas par exemple de lignées de lapins histocompatibles qui présentent un intérêt pour les études d'immunopathologie et pour l'étude fondamentale du complexe majeur d'histocompatibilité (CMH) dont la structure est plus proche de celle de l'homme que ne l'est par exemple celle de la souris (Marche et al, 1989; Laverrière et al, 1989).

C'est aussi le cas pour la lignée que nous avons choisie, qui est constituée par des animaux sélectionnés par le Dr A Kelus (depuis de nombreuses années à l'Institut d'immunologie de Bâle) sur la variabilité allotypique des gènes codant pour la production des immunoglobulines. Les animaux de chaque allotype sont très homogènes entre eux (Mage et al, 1982; Knight et al, 1985). En 1990, une vingtaine d'individus représentant 3 allotypes différents, alibas (souche Kelus), $3 \mathrm{R} 4 \mathrm{~K}$ bas et $2 \mathrm{H} 4 \mathrm{G} 4 \mathrm{~F}$ ont servi de fondateurs pour 3 familles maintenues à la station de pathologie expérimentale de l'INRA de Nouzilly ( $\mathrm{P}$ Coudert, données non publiées). Le taux de consanguinité de ces familles est maintenant élevé et la prolificité des femelles est faible, de 0 à 3 lapereaux par portée; le coût d'entretien d'un tel troupeau est trop élevé pour une demande ponctuelle. L'objectif de notre travail est donc de conserver ces familles uniquement sous forme d'embryons et de semence congelés.

Vingt-deux femelles ont été accouplées avec 11 mâles de même allotype puis sacrifiées afin de congeler leurs embryons. En complément, la semence de 8 mâles a été congelée. Un total de 264 embryons et 137 paillettes de semence représentant les 3 souches de la lignée est maintenant mis à l'abri. Ces souches sont ainsi complètement conservées $e x$ situ. 


\section{Une lignée issue d'une mutation monogénique : le lapin dit «sauteur d'Alfort»}

Contrairement à la situation qui prévaut chez la souris, les lignées caractérisées par l'effet d'un seul gène sont peu nombreuses. Il y a peu de mutations monogéniques naturelles isolées (Letard, 1935) et le nombre de lignées transgéniques quoiqu'en augmentation rapide est encore faible (Massoud et al, 1991; Aigner et al, 1993) .

Nous avons choisi comme exemple une lignée issue d'une mutation spontanée, celle dite du «lapin sauteur d'Alfort». La particularité de cette population se manifeste par un comportement locomoteur original : l'animal se déplace sur les membres antérieurs en soulevant son arrière train à la verticale de sa tête; il fait le «poirier». Ce caractère monogénique récessif apparu à la suite d'une mutation spontanée (Letard, 1935) est associé chez la plupart des animaux à une cataracte (pénétrance variable). La souche présente donc un double intérêt : c'est un modèle possible pour la microchirurgie oculaire et pour la sémiologie neurologique.

Actuellement, cette population comprend plusieurs dizaines d'individus conservés in situ dans 6 élevages agréés permettant la fourniture de sujets pour la recherche (Boucher, 1991). L'intérêt de la cryobanque est de préserver ex situ ce gène original. L'échantillon est donc constitué d'un seul couple d'animaux homozygotes pour ce gène et leurs descendants sont utilisés comme donneurs d'embryons. Seules quelques dizaines d'embryons congelés suffisent pour sauvegarder cette mutation.

\section{PERSPECTIVES}

Le programme de cryopréservation est actuellement étendu à des lignées transgéniques (Massoud et al, 1991) et à des groupes particuliers de reproducteurs. Ces derniers sont constitués par des clônes d'embryons, c'est-à-dire des lots d'embryons de même constitution génétique obtenus par transfert des noyaux d'un embryon donneur au stade morula (environ 64 noyaux) dans autant d'ovocytes receveurs énucléés. Ces embryons peuvent après transplantation dans une femelle porteuse donner naissance à des jeunes viables et normalement fertiles (Heyman et al, 1990). Les taux de réussite sont encore faibles puisque seulement 5 à $10 \%$ d'entre eux se développent à terme. Mais on peut anticiper une meilleure maîtrise de cette technologie sophistiquée dans les prochaines années, ce qui justifie déjà la mise à l'abri, dans une cryobanque, de petits lots représentatifs de ce type d' embryons.

Les embryons et spermatozoïdes d'autres races dont le choix est actuellement à l'étude devraient prochainement être à leur tour pris en compte. Il deviendra alors possible d'examiner l'effet du génotype des embryons sur leur taux de survie après décongélation. En effet, les embryons de lapin manifestent apparemment, selon leur origine génétique, des différences de sensibilité aux processus de congélationdécongélation (Vicente et Garcia Ximenez, 1993). Ces différences sont aussi mises en évidence chez la souris (Pomp et Eisen, 1990). Chez cette espèce toutefois, les taux de survie après décongélation ne varient entre eux que de $35 \%$ ce qui amène à ne prendre en compte ces données que pour ajuster la taille de l'échantillon.

Les résultats acquis chez le lapin pourront alors être utilisés pour définir les bases de l'économie d'un plan rationnel de conservation du patrimoine génétique d'une espèce domestique à l'instar de ce qui est en cours de réalisation pour plusieurs 
populations végétales (Joly et Trommeter, 1991). À terme, l'introduction dans la banque de lignées de cellules totipotentes congelées devra être considérée. Ces cellules dites ES (embryonic stem cells) ou EG (embryonic germinal cells) sont de plus en plus utilisées chez la souris car elles présentent l'intérêt de pouvoir participer à la formation de la lignée germinale au sein de chimères d'agrégation, et donc de voir leurs caractères transmis aux descendants, notamment après introduction ou remplacement ciblé d'une séquence génique donnée par recombinaison homologue. Les résultats chez le lapin sont toutefois encore très limités (Moens et al, 1992).

L'intérêt d'une banque se mesure aux échanges qu'elle permet entre différents partenaires. Des modalités de fonctionnement impliquant des relations contractuelles entre laboratoires de recherche et éleveurs et prenant en compte les conséquences des décisions concernant la brevetabilité éventuelle des lignées animales (Milligan et Lesser, 1989; Hermitte et Joly, 1991) sont actuellement expérimentées à l'échelon national. L'élargissement à d'autres pays va constituer une deuxième étape qui sera engagée à travers une action concertée de la commission des Communautés européennes soutenue par la direction générale pour la science, la recherche, et le développement (DG XII, programme PL 920184). Une façon de montrer que les responsables des ressources génétiques des pays d'Europe doivent rapidement et concrètement se rassembler. Mais aussi un début de réponse à l'ambitieux programme de banques d'embryons de souris et de lapin mis actuellement en place aux États-Unis (Gershon, 1993) par l'Institut national de la santé (NIH), banques qui pourraient constituer un premier pas pour d'autres espèces animales.

\section{REMERCIEMENTS}

Nous tenons à remercier $\mathrm{G}$ Arnold et les responsables de la Fédération française de cuniculture pour l'intérêt qu'ils portent à cette recherche. Nous remercions aussi B Poujardieu pour ses conseils lors de la préparation de ce document. Cette recherche, initiée en 1991, a été financée par l'INRA dans le cadre de son action Agrotech (programme Prodige) et par la Fondation pour la recherche médicale (FRM) qui nous permet aujourd'hui de poursuivre notre travail.

\section{RÉFÉRENCES}

Aigner B, Brem G (1993) Tyrosinase as a marker gene and model for screening transgenes in mice and rabbits. Theriogenology 39,177

Bolet G, de Rochambeau H, Coudert P (1991) Caractéristiques génétiques des souches de lapins de l'INRA. In : $11^{\text {es }}$ Journées d'études IFFA CREDO. Marcyl'Étoile, France, 3-4 octobre, Fondation Mérieux, Lyon, 91, 35-52

Boucher S (1991) Le lapin sauteur d'Alfort. Rev-Avicole 3, 91-95

Brem G, Graf F, Krausslich H (1982) Möglichkeiten der anlage von genreserver. Genetische probleme und kostete. Bayer Landr Jb 59, 380-383

Courot M (1977) L'insémination artificielle chez les lapins. Cuniculture 4, 135-136 FAO (1987) Definitions pertaining to animal genetic resources. In : anim Genet resources (Hodges $\mathrm{J}$, ed) FAO Animal Production and Health paper $\mathrm{n}^{\circ} 66$, appendix 6,315 
Garnier V, Renard JP, Menezo Y (1988) Viability and freezing ability of rabbit embryos collected in the vagina after prostaglandin treatment. Jpn J Physiol 38, 585-589

Gershon D (1993) NIH to support embryo bank for mice and rabbits. Nature 362, 684

Glenister PH, Whittingham DG, Lyon MF (1984) Further studies on the effect of radiation during the storage of frozen 8-cell mouse embryos at $-196^{\circ} \mathrm{C}$. $J$ Reprod Fert 70, 229-234

Heyman Y, Chesné P, Renard JP (1990) Reprogrammation complète de noyaux embryonnaires congelés, après transfert nucléaire chez le lapin. CR Acad Sci 311, Série III, 321-326

Hermitte MA, Joly PB (1991) Biotechnologie et brevets : un essai d'analyse économique. In: Innovation, changement technique et agroalimentaire. INRA Actes Communication 8, 67-82

Joly PB, Trommeter M (1991) World regulation of genetic resources: is the model of common heritage sustainable? In: Int Symp IFFAS, Nairobi, juin 1991

Kennelly JJ, Foote RH (1965) Superovulatory response of pre- and post-pubertal rabbits to commercialy available gonadotrophins. J Reprod Fert 9, 177-188

Knight KL, Burnett RC, Nicholas JM (1985) Organization and polymorphism of rabbit immunoglobulin heavy chain genes. $J$ Immunol 134, 1245-1250

Laverrière A, Kullaga H, Kindt TJ, Lequerse C, Marche PN (1989) A rabbit class II gene with strong similarities to HLA-DRA. Immunogenetics 30, 137-140

Letard E (1935) Une mutation nouvelle chez le lapin. Bull Acad Vét Fr 8, 608-610 Lukefahr SD (1985) A note on an estimate of the World's domestic rabbit population. J Appl Rabbit Res 8, 157

Lukefahr SD (1990) Conservation of global rabbit germplasm resources. $J$ Appl Rabbit Res 12, 283-290

Mage RG, Dray S, Gilman-Sach A et al (1982) Rabbit heavy chair haplotypesallotypic determinants expressed by $\mathrm{V}_{\mathrm{h}}-\mathrm{C}_{\mathrm{h}}$ recombinants. Immunogenetics 15 , 287297

Maijala K (1984) Conservation of animal genetic resources in Europe. Livest Prod Sci 11,3

Marche PN, Rebirre MC, Leguerse C, Kindt TJ (1989) Structure and expression of rabbit MCH genes. Ann Rech Vét 20, 361-365

Massoud M, Bischoff R, Dalemans W et al (1991) Expression of active recombinant human a $a_{1}$-antitrypsine in transgenic rabbits. $J$ Biotech 18, 193-204

Matheron G, Chevalet B (1977) Conduite d'une population témoin de lapin : évolution à court terme du coefficient de consanguinité selon le schéma d'accouplement. Ann Génét Sél Anim 9, 1-13

Matheron G, Poujardieu B (1984) La génétique du lapin : le point, les perspectives. In : Proc III World Rabbit Congress. Rome, 4-8 avril 1984, WRSA, 1, 3-32

Mazur P (1990) Equilibrium, quasi-equilibrium, and nonequilibrium freezing of mammalian embryos. Cell Biophys 17, 53-92

Milligan R, Lesser W (1989) Animal patents implication for agriculture. In : Animal patents (Lesser W, ed). Stockton Press, New-York ,100-113

Moens A, Fléchon JE, Renard JP (1992) Progress in the culture of rabbit embryonic cells. In : 12th ICAR. La Haye, 23-24 août 1992, ICAR, 2, 721-723 
Pomp D, Eisen EJ (1990) Genetic control of survival of frozen mouse embryos. Biol Reprod 42, 775-786

Renard JP, Rochambeau H de, Lauvergne JJ (1983) Utilisation of gametes and embryos banking for the preservation and study of genetic resources in farm animals. In : Proc World Conf Anim Prod, Tokyo, 1, 66-72

Renard JP, Bui Xuan-Nguyen, Garnier V (1984) Two strop freezing of two cell rabbit embryos after partial dehydration at room temperature. $J$ Reprod Fert 71 , 573-580

Rochambeau H de, Vrillon JL (1980) Faut-il sauver nos races de lapins? Cuniculture 7, 103-105

Rochambeau $\mathrm{H}$ de, Chevalet $\mathrm{C}$ (1989) Aspects théoriques de la gestion des ressources génétiques : cas des populations en conservation. In : La gestion des ressources génétiques des espèces animales domestiques (BRG, éd). Tec-Doc Lavoisier, Paris, $171-180$

Smith CH (1984) Genetic aspects of conservation in farm livestock. Livest Prod Sci $11,37-48$

Theau-Clément M, Vrillon JL (1989) Le point sur l'insémination artificielle. Cuniculture 16, 141-149

Theau-Clément M, Roustan A (1991) L'insémination chez la lapine. Elevage et Insémination 245, 3-12

Vicente JS, Garcia-Ximénez F (1993) Effects of strain and embryo transfer model (embryos from one versus two donor does/recipient) on results of cryopreservation in rabbit. Reprod Nutr Dev 33, 5-13

Wittingham DG, Adams CE (1976) Low temperature preservation of rabbit embryos. J Reprod Fert 47, 269-274

Wood MJ , Whittingham DG, Rall WF (1987) The low temperature preservation of mouse oocytes and embryos. In : Mammalian development a practical approach (Monk M, ed). IRL Press, 255-280

Woodford MH (1990) Cryogenic preservation of wild animal germplasm. In : Animal genetic resources : a global program for sustainable development (Wiener, ed). FAO Animal Production and Health paper, Rome, vol 80, 69-82

Yamada Y, Kimura K (1984) Survival probability in small livestock populations. In : Animal genetic resources conservation by management, data banks and training (Wiener, ed). Rome, FAO Animal Production and Health paper, vol 44, 105-110 